\title{
ANTIOXIDANT ACTIVITIES OF ROPINIROLE AND PRAMIPEXOLE NOVEL DRUGS USED IN TREATMENT OF PARKINSONISM: AN IN VITRO APPROACH
}

\author{
PREETHA SELVA*, SRINIVASAN V
}

Department of Pharmacology, Saveetha Medical College \& Hospital, Chennai, Tamil Nadu, India. Email: drpreethaselva@gmail.com Received: 24 August 2016, Revised and Accepted: 30 August 2016

\section{ABSTRACT}

Objective: The objective of this study is to evaluate and compare the free radical scavenging activities of selective dopamine agonist namely ropinirole and pramipexole.

Methods: The antioxidant activity of ropinirole and pramipexole at various concentrations was done by 1, 1-diphenyl-2picrylhydrazyl (DPPH) free radical scavenging assay comparing it with ascorbic acid which was taken as standard.

Results: The free radical scavenging property as measured by DPPH method showed that ropinirole and pramipexole have got a potent free radical scavenging activity with that of ascorbic acid.

Conclusion: Novel drugs such as pramipexole and ropinirole are promising molecules in the field of oxidative damage related neurodegenerative disorders providing us an optimistic targeted approach toward neuroprotection.

Keywords: Free radical scavenging, 1, 1-diphenyl-2picrylhydrazyl assay, Anti-Parkinson's, Dopamine agonist.

(C) 2016 The Authors. Published by Innovare Academic Sciences Pvt Ltd. This is an open access article under the CC BY license (http://creativecommons. org/licenses/by/4. 0/) DOI: http://dx.doi.org/10.22159/ajpcr.2016.v9s3.14861

\section{INTRODUCTION}

Parkinson's disease is a slowly progressing neurodegenerative disorder characterized by bradykinesia (slowing of movements), rigidity, increased muscle tone, tremors, and postural instability which is mainly due to loss or depletion of dopaminergic neurons in the substantia nigra [1]. The cause of this disease is multifactorial although the exact mechanism is still unknown. Recent studies have suggested that oxidative stress and abnormalities in the mitochondria can lead to increased free radical formation which might contribute to the pathogenesis of Parkinson's disease [2]. Administration of an exogenous neurotoxin called MPTP is also said to cause this disease due to selective loss of dopaminergic neurons in substantia nigra [3]. The main aim of drug therapy in Parkinsonism is to provide symptomatic relief to the patient by use of drugs that can restore the dopaminergic and cholinergic balance that is disturbed in this case. Dopamine itself cannot be given as it cannot cross the blood-brain barrier (BBB), and its peripheral effects have no benefits in Parkinsonism patients. Hence, levodopa - the precursor of dopamine - is given, which can cross the BBB and get decarboxylated to dopamine on entering the brain. However dopamine, which is formed from exogenously administered levodopa, is reported to be neurotoxic due to its conversion to some toxic products such as quinones and other free radicals [4,5]. These free radicals released might further increase the oxidative stress contributing to the pathogenesis of Parkinson's disease. Moreover, patients on long-term levodopa therapy experience a lot of adverse effects such as "on-off" phenomenon, dyskinesias, wearing off phenomenon, hallucinations, delusions, sleep disturbances, and schizophrenia-like symptoms [6]. These shortcomings of levodopa lead to the need for newer therapeutic agents with a neuroprotective role in the treatment of Parkinson's disease [7]. Now, dopamine agonists such as bromocriptine, ropinirole, pergolide, pramipexole, cabergoline, and rotigotine have said to have a neuroprotective effect in experiments conducted on animal models [8]. They have also reported to prevent the clinical progression of disease. Studies have shown that ergot derivatives of dopamine agonists such as bromocriptine and cabergoline have free radical scavenging property and possess antioxidant activity in vivo and in vitro. However, the main disadvantage of the ergot derivatives is increase in adverse effects such as anorexia, nausea, vomiting, vertigo, postural hypotension, painless peripheral vasospasm (spasm of digits), peripheral edema, pleural fibrosis, and erythromelegia [9]. These ergot derivatives also have poor tolerability profile. These drawbacks of ergot derivatives are overcome by non-ergot derivatives such as ropinirole and pramipexole which have fewer side effects and better tolerability profile [10]. However, unlike the ergot dopamine agonists, very few studies have reported the neuroprotective role of non-ergot derivatives of dopamine agonists such as ropinirole, pramipexole, and talipexole. The neuroprotective role of these non-ergot dopamine agonists is also probably because of its free radical scavenging and antioxidant activity. Hence, this study was undertaken to evaluate the antioxidant and free radical scavenging activity of non-ergot dopamine agonists - ropinirole and pramipexole, novel agents used in the treatment of Parkinson's disease using the in vitro 1,1-diphenyl-2picrylhydrazyl (DPPH) assay.

\section{Objective}

The objective of the present study was to evaluate and compare the antioxidant potential and free radical scavenging activity of ropinirole and pramipexole - non-ergot dopamine agonists used in the treatment of Parkinsonism with that of standard (ascorbic acid) using the in-vitro DPPH assay.

\section{METHODS}

Chemicals

DPPH and drugs pramipexole and ropinirole were obtained from the Sigma Chemical Co. CRC, Bangalore. Ascorbic acid was obtained from the SD Fine-Chem. Limited, Biosar, India.

\section{DPPH free radical scavenging activity}

DPPH radical scavenging activity was done using the method of Brand-Williams et al. (1995) [11-13]. The DPPH assay is based on the reduction of DPPH a stable free radical. The DPPH solution was prepared $(0.004 \% \mathrm{w} / \mathrm{v})$ in $95 \%$ ethanol. The reaction mixture containing $1 \mathrm{ml}$ of DPPH solution ( $1 \mathrm{mM}$ in ethanol) with $3 \mathrm{ml}$ of different concentrations of ropinirole $(50,40,30,20,10$, and $5 \mu \mathrm{g} / \mathrm{ml})$ in ethanol was shaken and incubated in dark for 20 minutes at room temperature. Ropinirole was soluble in ethanol, and its various concentrations were prepared by dilution method. The resultant absorbance was recorded at 
$517 \mathrm{~nm}$ using a spectrophotometer (HACH 4000 DU ultraviolet-visible spectrophotometer). This procedure was similarly repeated for different concentrations of pramipexole $(50,40,30,20,10$, and $5 \mu \mathrm{g} / \mathrm{ml})$, and the resultant absorbance was recorded at $517 \mathrm{~nm}$. Ascorbic acid was used as the reference standard and dissolved in distilled water to make the stock solution with same concentration $(100 \mu \mathrm{g} / \mathrm{ml})$. Control sample was prepared containing the same volume. $95 \%$ ethanol served as blank. The percentage of DPPH free radical scavenging was calculated using the formula:

Scavenging $\%=\frac{\text { Ac-Asor At }}{\text { Ac }} \times 100$

Where, Ac is absorbance of control and As or At is the absorbance of standard or test. The inhibitory concentration $\left(\mathrm{IC}_{50}\right)$ value was calculated using Microsoft excels 2016. A scatter graph was made in excel, and the slope equation $\mathrm{Y}=\mathrm{a} * \mathrm{X}+\mathrm{b}$ was obtained. With this equation, the $\mathrm{IC}_{50}$ was calculated using the formula $\mathrm{IC}_{50}=(0.5-\mathrm{b}) / \mathrm{a}$.

\section{RESULTS}

At concentrations of $5,10,20,30,40$, and $50 \mu \mathrm{g} / \mathrm{ml}$ absorbance observed with ropinirole was $0.2256,0.1486,0.0869,0.0745,0.0629$, and 0.0527 , respectively. At the same concentrations, the absorbance observed with pramipexole was $0.2216,0.1239,0.0745,0.0648,0.0365$, and 0.0294 , respectively. Ascorbic acid which was the reference standard showed the absorbance of $0.1916,0.1182,0.0416,0.0391,0.0335$, and 0.0261 .

At concentrations of $5,10,20,30,40$, and $50 \mu \mathrm{g} / \mathrm{ml}$, the percentage of inhibition of DPPH free radical activity observed with ropinirole was $7.66 \%, 39.22 \%, 64.46 \%, 69.54 \%, 74.24 \%$, and $78.44 \%$, respectively (Table 1). At the same concentrations, the percentage of inhibition observed with pramipexole was $9.33 \%, 49.32 \%, 69.55 \%, 73.49 \%$, $85.07 \%$, and $88.01 \%$, respectively (Table 1). Ascorbic acid which was the reference standard had the percentage of inhibition of $21.61 \%$, $51.66 \%, 83.01 \%, 84.02 \%, 86.32 \%$, and $89.34 \%$ (Table 1). The $\mathrm{IC}_{50}$ value calculated was $16.29,18.19$, and $10.16 \mu \mathrm{g} / \mathrm{ml}$ for ropinirole, pramipexole, and ascorbic acid, respectively (Fig. 1-3).

\section{DISCUSSION}

Studies conducted earlier by Hauser et al., Gaki and Papavassiliou, and Niranjan $[2,14,15]$ have shown that increased oxidative stress and free radical formation contributes to the pathogenesis of Parkinson's disease. Hence, drug molecules which have free radical scavenging property will have the neuroprotective effect in patients with Parkinson's disease. From our study, the free radical scavenging property as measured by DPPH method showed that ropinirole and pramipexole have got an effective free radical scavenging activity when compared with ascorbic acid which was used as a standard (Fig. 4). The results of this study are analogous with the studies conducted earlier by Joyce et al., Park et al., and Tanaka et al. [16-18]. Hence, from our study and other studies conducted earlier, it is evident that even non-ergot derivatives such as pramipexole and ropinirole might have additional beneficial effects in the disease because of its antioxidant activity apart from acting as selective D2/D3 dopamine receptor agonists.

\section{CONCLUSION}

In this study, both ropinirole and pramipexole showed increasing free radical scavenging activity with increasing concentrations. Among the two drugs, pramipexole and ropinirole which were subjected to in-vitro DPPH

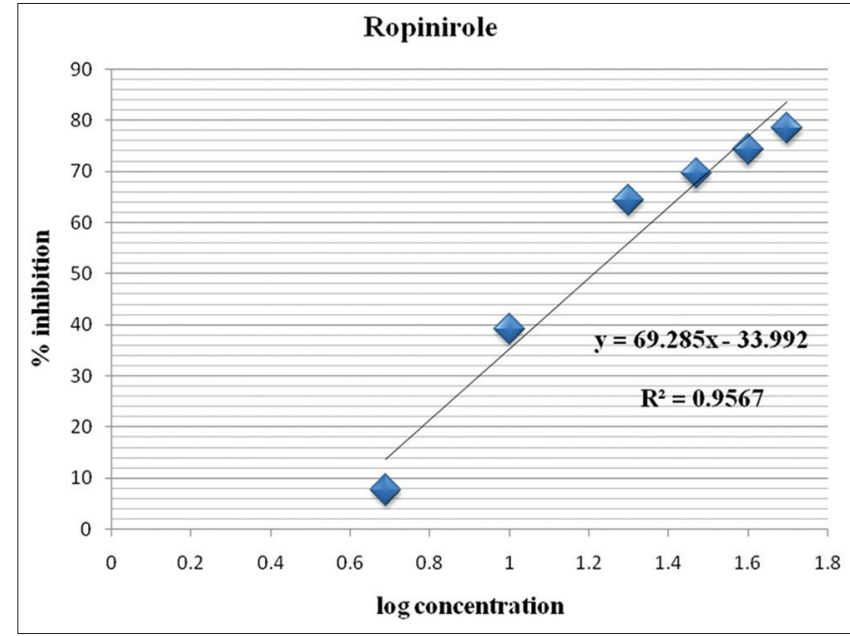

Fig. 1: Determination of inhibitory concentration 50 of ropinirole using linear regression

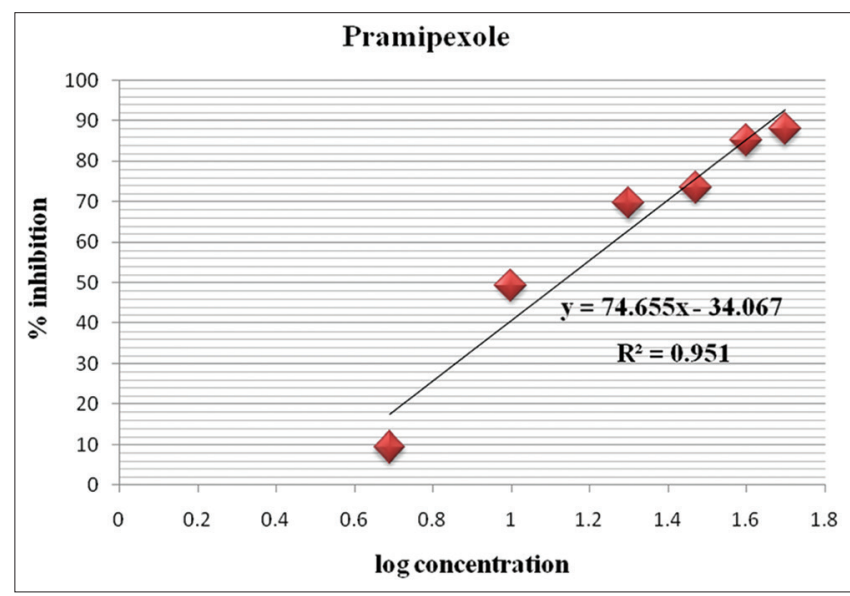

Fig. 2: Determination of inhibitory concentration 50 of pramipexole using linear regression

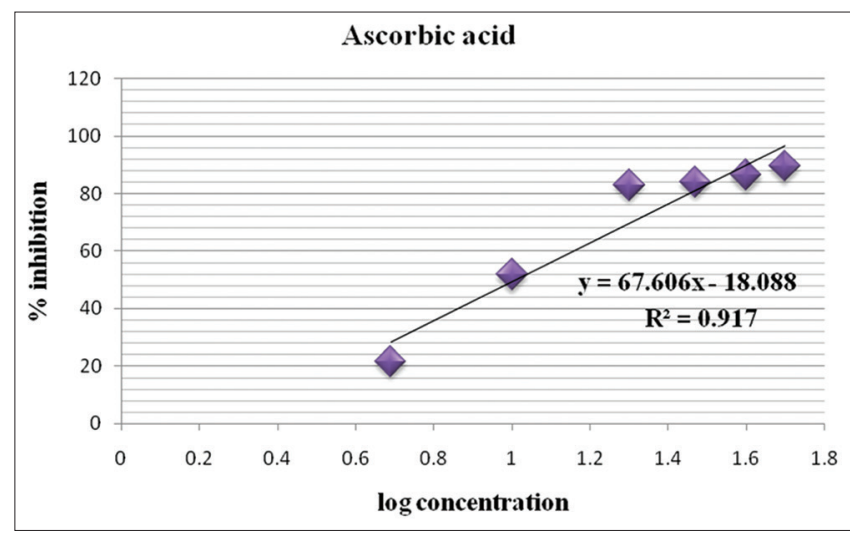

Fig. 3: Determination of inhibitory concentration 50 of ascorbic acid using linear regression

Table 1: Percentage of inhibition of ropinirole, pramipexole, and ascorbic acid at various concentrations

\begin{tabular}{llll}
\hline Compound concentration $(\boldsymbol{\mu g} / \mathbf{m l})$ & Ropinirole $(\%$ inhibition) & Pramipexole $(\%$ inhibition) & Ascorbic acid (\% inhibition) \\
\hline 5 & 7.66 & 9.33 & 21.61 \\
10 & 39.22 & 49.32 & 51.66 \\
20 & 64.46 & 69.55 & 83.01 \\
30 & 69.54 & 73.49 & 84.02 \\
40 & 74.24 & 85.07 & 86.32 \\
50 & 78.44 & 88.01 & 89.34 \\
\hline
\end{tabular}




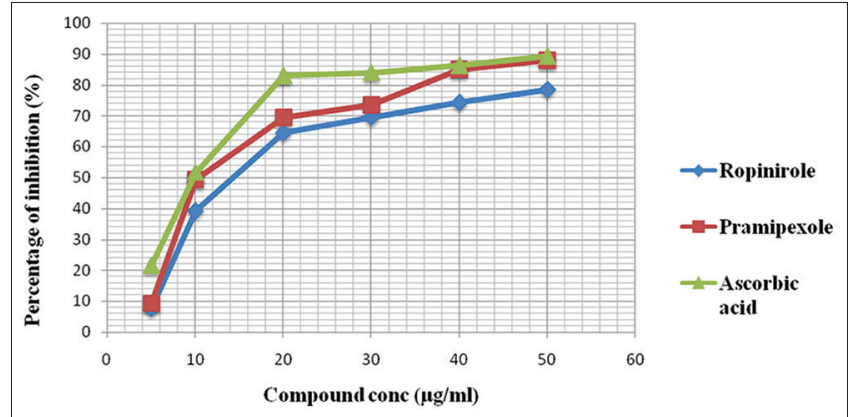

Fig. 4: 1, 1-diphenyl-2picrylhydrazyl radical scavenging activity of ropinirole, pramipexole, and ascorbic acid

assay, pramipexole showed the slightly higher percentage of inhibition when compared to ropinirole at all concentrations. However, the $\mathrm{IC}_{50}$ levels of both the drugs were almost similar. Hence, this study shows that pramipexole and ropinirole have got good free radical scavenging activity and is non-inferior to each other and could play a role of neoadjuvant antioxidant in a wide variety of neurodegenerative disorders.

\section{REFERENCES}

1. Schapira AH, McDermott MP, Barone P, Comella CL, Albrecht S, Hsu HH, et al. Pramipexole in patients with early Parkinson's disease (PROUD): A randomised delayed-start trial. Lancet Neurol 2013;12(8):747-55

2. Niranjan R. The role of inflammatory and oxidative stress mechanisms in the pathogenesis of Parkinson's disease: Focus on astrocytes. Mol Neurobiol 2014;49(1):28-38.

3. Pain S, Gochard A, Bodard S, Gulhan Z, Prunier-Aesch C, Chalon S. Toxicity of MPTP on neurotransmission in three mouse models of Parkinson's disease. Exp Toxicol Pathol 2013;65(5):689-94.

4. Borah A, Mohanakumar KP. L-DOPA induced-endogenous 6-hydroxydopamine is the cause of aggravated dopaminergic neurodegeneration in Parkinson's disease patients. Med Hypotheses 2012;79(2):271-3
5. Hattoria N, Wanga M, Taka H, Fujimura T, Yoritaka A, Kubo S, et al. Toxic effects of dopamine metabolism in Parkinson's disease. Parkinsonism Relat Disord 2009;15 Suppl 1:S35-8.

6. Jankovic J. Motor fluctuations and dyskinesias in Parkinson's disease: Clinical manifestations. Mov Disord 2005;20 Suppl 11:S11-6.

7. Bonuccelli U, Pavese N. Dopamine agonists in the treatment of Parkinson's disease. Expert Rev Neurother 2006;6(1):81-9.

8. Le WD, Jankovic J. Are dopamine receptor agonists neuroprotective in Parkinson's disease? Drugs Aging 2001;18(6):389-96.

9. Tintner R, Manian P, Gauthier P, Jankovic J. Pleuropulmonary fibrosis after long-term treatment with the dopamine agonist pergolide for Parkinson Disease. Arch Neurol 2005;62(8):1290-5.

10. Gottwald MD, Bainbridge JL, Dowling GA, Aminoff MJ, Alldredge BK. New pharmacotherapy for Parkinson's disease. Ann Pharmacother 1997;31(10):1205-17.

11. Brand-Williams W, Cuvelier ME, Berset C. Use of free radical method to evaluate antioxidant activity. Lebensm Wiss Technol 1995;28:25-30

12. EA N, Mathew L. A comparative evaluation of antimicrobial and antioxidant properties of Thevetia neriifolia, juss fruit rind extracts. Int J Curr Pharm Res 2014;6(2):47-50.

13. Sakat S, Juvekar AR, Gambhire MN. In vitro antioxidant and antiinflammatory activity of methanol extract of Oxalis corniculata Linn. Int J Pharm Pharm Sci 2010;2(1):146-55.

14. Hauser DN, Hastings TG. Mitochondrial dysfunction and oxidative stress in Parkinson's disease and monogenic Parkinsonism. Neurobiol Dis 2013;51:35-42

15. Gaki GS, Papavassiliou AG. Oxidative stress-induced signaling pathways implicated in the pathogenesis of Parkinson's disease. Neuromolecular Med 2014;16(2):217-30.

16. Joyce JN, Woolsey C, Ryoo H, Borwege S, Hagner D. Low dose pramipexole is neuroprotective in the MPTP mouse model of Parkinson's disease, and downregulates the dopamine transporter via the D3 receptor. BMC Biol 2004;2:22.

17. Park G, Park YJ, Yang HO, Oh MS. Ropinirole protects against 1-methyl-4-phenyl-1, 2, 3, 6-tetrahydropyridine (MPTP)-induced neurotoxicity in mice via anti-apoptotic mechanism. Pharmacol Biochem Behav 2013;104:163-8.

18. Tanaka K, Miyazaki I, Fujita N, Haque ME, Asanuma M, Ogawa N. Molecular mechanism in activation of glutathione system by ropinirole, a selective dopamine D2 agonist. Neurochem Res 2001;26(1):31-6. 